\title{
No Sib Pair Concordance for Breast or Ovarian Cancer in BRCAI Mutation Carriers
}

\author{
Pål Møller', Lovise Mæhle', Neal Clark', Jaran Apold² \\ ISection for Inherited Cancer, Department of Medical Genetics, Rikshospitalet-Radiumhospitalet Medical Center Oslo, Norway \\ 2Department of Medical Genetics, Haukeland University Hospital, Bergen, Norway
}

Key words: BRCAl, modifiers, expression, genetic counselling

Corresponding author: Pål Møller, M.D. Ph.D., Section for Inherited Cancer, The Norwegian Radium Hospital, N-0310 0slo, Norway, e-mail: pmoller@ulrik.uio.no

Submitted: 13 February 2007

Accepted: 7 May 2007

\begin{abstract}
Modifying factors might theoretically determine whether a BRCA1 mutation carrier contracts breast or ovarian cancer. If so, one would expect concordance for breast or ovarian cancer in affected sibships. We identified 64 pairs with cancers where one or both sisters were demonstrated to carry a BRCA 1 mutation, and 116 additional constructed pairs in sibships with three or more affected sisters. We analysed concordance for breast and for ovarian cancer both in the complete series and in the 64 sister pairs alone. The results were that concordance for both breast and ovarian cancer in sisters was in keeping with random distribution or multiple and frequent modifying genetic factors. In conclusion, there may be no major modifying factor of expression of BRCA 1 mutations. The practical implication of our findings is that previous disease manifestations in close relatives may have no bearing on the first cancer to be expected in a young female mutation carrier.
\end{abstract}

\section{Introduction}

Breast and ovarian cancers are both expressions of BRCAl germ-line mutations. There have been speculations whether or not modifying factors may determine whether a mutation carrier contracts breast or ovarian cancer. There are reports on environmental factors having such effects in the population and/or in BRCA1 mutation carriers (oral contraceptives, etc.) [1-2], natural variations in sex hormones (childbirth, etc.) [3], and also lifestyle [4]. Germline BRCA1 mutations (OMIM + 113705) cause breast and/or ovarian cancer. Besides sex and highly penetrant mutations, there are no described major predictor factors determining who is to contract breast cancer, or when she might do so. Whether the position of the mutation within each gene is indicative of what kind of cancer a BRCA mutation carrier may contract has been discussed [5-7].
The possibility of genetic modifiers of expression of $B R C A 1$ mutations is important for three reasons:

1. Does a BRCA1 mutation carrying sister or daughter to an ovarian or breast cancer patient have higher risk for ovarian or breast cancer, respectively, than other BRCA1 mutation carrying women? Such questions are frequently asked during genetic counselling. Because health care with respect to oophorectomy and mastectomy is a personal choice, lack of evidence may lead to intervention based on unsubstantiated fear. Besides the obvious problems with decision making in the absence of knowledge, the fear itself may be considered a health problem.

2. The other question is cost/benefit of research programmes to look for modifiers of penetrance or expression of BRCA1 mutations. 
3. The third question is whether the family history may indicate which mutations to look for when performing mutational analyses in a new family.

Also, if there are environmental factors influencing BRCAl expression, they should be present and subject to identification within concordant pairs. If so, this may be a way to identify behaviour patterns to avoid contracting breast or ovarian cancer.

We addressed these questions by examining our BRCA1 mutation carrying kindreds for whether there was an association between the expression of the mutations between sisters. If an association could be demonstrated, there might be single modifiers determining which cancer a mutation carrying woman may contract. If not, there may not be modifiers of clinical significance to predict which expression of a BRCA1 mutation a given woman is likely to contract.

\section{Material and methods}

BRCA 1 genetic variants including frameshift, stop and large deletions/insertions were scored as "mutations". Most of the Norwegian BRCA1 mutation carriers have one out of a few frequent mutations [8-9]. We arranged high capacity testing for the frequent mutations and expanded the pedigrees for all new mutation carriers demonstrated. All diagnoses in the families were verified in hospitals' medical files or in the Norwegian Cancer Registry. We offered all families predictive testing. Information flow in the family was good, and uptake of testing about 80\% [10]. All mutation carriers were offered follow-up examinations aiming at early diagnosis and treatment, which apart from a marginal personal fee is paid by the state insurance. All activities were health services, all women were given genetic counselling and all testing was subject to written informed consent according to national legislation. All confirmation of diagnoses in medical files or cancer registry was subject to written informed consent. All BRCAl mutation carrying kindreds were included. For all members of any kindred, we record mother's and father's identity within the pedigree structure, allowing us to identify all sister pairs. All information was kept and analysed in our computerized medical files (application written in $\mathrm{dB}+$, data kept in Oracle (C) format), analysed by means of TOAD (C) and no research registry was created.

We identified all female BRCA1 mutation carriers who had or had had one or more sisters in our medical files and verified all their diagnoses. To avoid distorted results because of prophylactic oophorectomy recently advocated to mutation carriers, we censored all data at the end of 2004.
BRCA1 mutation carriers frequently contract more than one primary cancer - they may demonstrate bilateral breast cancer and/or both breast and ovarian cancer. Because the probability of developing more than one cancer is influenced by the probability of surviving the first one, and because treatment given to cure the first cancer may influence the probability of contracting a second primary, we decided to score primary cancers only. Each woman was scored as having breast or ovarian cancer as her first cancer. All but one of those selected for the present study were registered as having breast or ovarian cancer as the first or only cancer. In one woman, breast cancer was diagnosed when she was initially treated for ovarian cancer. She was scored as having ovarian cancer.

Both breast and ovarian cancers may be lethal, and their prognosis may differ. All available affected sisters were tested. We did not have access to testing of deceased patients, and included all sibships where one or more of the affecteds had a demonstrated BRCA 1 mutation. Obviously, affected pairs demonstrated to be discordant for BRCA1 mutations were excluded.

In some families, we identified sibships with three or more affected sisters. A triplet gives three possible pairs, etc. We analysed the material twice:

- Initially, we analysed all affected pairs, ignoring all triplets and quadruplets. Such an analysis will reduce sample size and power, but may not disturb outcome if a modifying factor had no association with numbers of daughters.

- Thereafter, we repeated the analyses also including all possible pairs within affected triplets and quadruplets. In principle, this may give factors inside triplets and quadruplets more weight in the analyses because each person was counted more than once.

Expected numbers of breast-breast and ovarian-ovarian cancer pairs were calculated as the square of the prevalence of each cancer in the total series multiplied by the number of pairs in the series. Expected numbers of breast-ovarian/ovarian-breast cancer pairs were calculated as twice the product of the prevalence of breast cancers multiplied by prevalence of ovarian cancers in the series and multiplied by the number of pairs in the series.

\section{Results}

\section{Pairs}

We identified 64 pairs with breast or ovarian cancers as first cancers in sibships with two affecteds, and where at least one of the affecteds had a demonstrated BRCA1 mutation (Table 1). 
Table 1. Sibships with two affected sisters. Number of patients with breast or ovarian cancer, calculated frequency, calculated expected pairs concordant for given phenotype assuming random distribution, observed number of concordant pairs, and results of test for independence

\begin{tabular}{|c|c|c|c|c|c|}
\hline Cancer & $\begin{array}{l}\text { Number } \\
\text { of patients }\end{array}$ & Frequency & Expected concordant pairs & $\begin{array}{c}\text { Observed } \\
\text { concordant pairs }\end{array}$ & Chi square $(p)$ \\
\hline breast & 78 & $78 / 128=0.61$ & $64 \times(0.61)^{2}=23.8$ & 22 & $0.13(>0.05)$ \\
\hline ovarian & 50 & $50 / 128=0.39$ & $64 \times(0.39)^{2}=9.8$ & 8 & $0.32(>0.05)$ \\
\hline
\end{tabular}

Table 2. As Table 1, but also including all constructed pairs from triplets and quadruplets

\begin{tabular}{lcccr}
\hline Cancer & $\begin{array}{c}\text { Number } \\
\text { of patients }\end{array}$ & Frequency & Expected concordant pairs & $\begin{array}{c}\text { Observed } \\
\text { concordant pairs }\end{array}$ \\
\hline breast & 209 & $209 / 360=0.58$ & $180 \times(0.58)^{2}=60.7$ & 63 \\
\hline ovarian & 151 & $151 / 360=0.42$ & $180 \times(0.42)^{2}=31.7$ & 34 \\
\hline
\end{tabular}

\section{Constructed pairs from triplets and quadruplets}

We identified 116 additional constructed pairs representing all possible pairs from affected triplets and quadruplets (Table 2).

As seen in Table 1 and Table 2, the expected numbers of concordant pairs closely met the observed numbers. Moreover, the observed numbers of concordant pairs were slightly less than expected when calculating for two-affected sibships only, which is opposite to the hypothesis tested that there may be an excess of concordant pairs.

\section{Discussion}

The results were in keeping with the interpretation that in BRCA1 mutation carriers, breast and ovarian cancer are independently occurring: whether two BRCAT mutation carrying sisters will contract the same or different kinds of cancer appears to be stochastic. If so, cancer type in a BRCA 7 mutation carrying woman may have no bearing on what is to be expected in her mutation carrying sister. Phenotypes of cancers previously registered in the kindred may have no bearing on what to expect in a healthy young mutation carrier. This interpretation has a direct bearing on genetic counselling sessions where BRCAl mutation carrying women have to decide what to do. This interpretation was derived directly from the observations and included no assumptions on the underlying mechanism(s).

Numbers were small, but possibly large enough to reveal any major factor. Autosomally, recessively inherited modifying factors are assumed to produce sib concordance without parent-offspring concordance. Autosomally, dominantly inherited modifiers are assumed to produce both parent-offspring and sib concordance. Multifactorial factors modifying penetrance (genetic and/or environmental) would be assumed to give more concordance between sibs than parent-offspring. In this way, both interaction caused by monogenic mechanism(s) as well as multifactorially caused influence(s) were assumed to produce sister correlation. The results were that no signs of such modifiers were seen.

Multiple factors with low penetrance of each factor may in analyses mimic random distributions. In real-life practice, however, this problem is of less importance, because the empirical observation without any assumption was that cancer type in one affected will not predict cancer type in her sister. In a scientific framework the problem is difficult to address: Multifactorial inheritance may not be described by genetic linkage, and if sister concordance appears random, affected sib pair approaches may be difficult as well. If concordance had been found, we would have used the concordant pairs to look for presence of modifying factors - the results give such a strategy lower probability for success.

The majority of the pairs had one of the frequent Nonvegian BRCA1 mutations. If some of these mutations carried risk for breast cancer only, and some for ovarian cancer only, concordance between pairs should have been observed. The lack of observed concordance indicates that the mutations causing cancers in the sibships carried no differences in expression with respect to breast and ovarian cancer. This leads to the notion that family history of distribution of breast and ovarian cancers may give no indication of which BRCA1 mutation 
to look for. Obviously, the number of frequent mutations examined was low, and to exclude a position effect as reported by others [5] it would have been beneficial to have a wider variation in frequently occurring mutations within the cohort examined.

There are a number of ascertainment and statistical problems in an analysis like this:

- We did not restrict the analyses to pairs where both sisters had a demonstrated BRCAl mutation, because breast and ovarian cancer may carry different prognoses. Concordance when both were alive and testable might reflect that one type of cancer has a better chance to survive.

- We did not analyse age at onset of disease, because early age at onset was a selection criterion to ascertain the families examined.

- As discussed initially, we did not consider second or third primary cancers.

- There is a possibility that an untested affected woman to a BRCA 1 carrier does not carry the mutation. We applied no correction for this probability. Because breast cancer is more frequent in the general population, a slight concordance for breast cancer might have been expected. On the other hand, ovarian cancer in the family may induce BRCA1 testing, and may artificially increase concordance for ovarian cancer also when the ovarian cancer patient was dead and not testable.

- Chemoprevention (tamoxifen, etc.) will invalidate a study like this. Chemoprevention for breast cancer is not allowed in Norway and had not been used in the cohort studied.

- Prophylactic oophorectomy and mastectomy may invalidate a study like this. The uptake of prophylactic mastectomy in Norway has been low. Until a few years ago we conducted a large observational study for early diagnosis of ovarian cancer and prophylactic oophorectomy was rarely performed, while most older mutation carriers today choose oophorectomy. In principle, this was a retrospective study; disturbances in outcome produced as an effect of our interventions in the last decade were marginal, because our intervention in principle was early detection and not chemoprevention or prophylactic surgery. We observed a total of no more than six breast cancers in BRCA 1 mutation carriers after oophorectomy, and no ovarian cancer in women who had had prophylactic mastectomy. Because we considered first cancer only, oophorectomy or mastectomy after first cancer will not affect the results.

We considered our material informative and probably not significantly influenced by confounders making such studies difficult. We may not wait to arrive at larger numbers included, because the series has now become invalidated by prophylactic surgery. Because we have high compliance, because we have documented close to all cancers in the families in the medical files and/or cancer registries, because our families are large, thereby minimizing the effect of calculation on the selection clusters in the families, because we have complete pedigree structures for close to all pedigrees in question, and because we know that we have tested many or all living affecteds in the present generations, we find that our findings support the notion that there may be no major modifying factor. Modifying factors - if they exist - may be multiple and frequently occurring. Common, low-penetrant genes causing familial breast cancer in the absence of BRCA1 or BRCA2, and simultaneously acting as modifiers of penetrance for $B R C A 1$ and $B R C A 2$, have been suggested [1 1 -12]. However, the reports do not indicate a similar volume of genes besides BRCA 1 and BRCA2 causing inherited ovarian cancer. Thus, to make the model fit our findings, such modifiers may produce breast cancer and not ovarian cancer in the absence of BRCA1 or BRCA2.

In conclusion, observed phenotypes in the family may not indicate which cancer a BRCA1 mutation carrier is at risk for and family history may give no clue as to which mutation to search for in breast-ovarian cancer kindreds. With respect to genetic counselling, this means that in kindreds with predominantly ovarian cancer, the cancer to consider in a young BRCA 1 carrier is breast cancer because breast cancer occurs at an earlier age. In BRCA1 mutation carrying kindreds with breast cancer alone, the risk for ovarian cancer may be as high as in ovarian cancer kindreds.

\section{References}

1. Narod SA, Risch H, Moslehi R, Dorum A, Neuhausen S, Olsson H, Provencher D, Radice P, Evans G, Bishop S, Brunet JS, Ponder BA. Oral contraceptives and the risk of hereditary ovarian cancer. Hereditary Ovarian Cancer Clinical Study Group. N Engl J Med 1998; 339: 424-428.

2. Narod SA, Dubé MP, Klijn J, Lubinski J, Lynch HT, Ghadirian P, Provencher D, Heimdal K, Moller P, Robson M, Offit K, Isaacs C, Weber B, Friedman E, Gershoni-Baruch R, Rennert G, Pasini B, Wagner T, Daly M, Garber JE, Neuhausen SL, Ainsworth P, Olsson H, Evans G, Osborne M, Couch F, Foulkes WD, Warner E, Kim-Sing C, Olopade O, Tung N, Saal HM, Weitzel J, Merajver S, Gauthier-Villars M, Jernstrom H, Sun P, Brunet JS. Oral contraceptives and the risk of breast cancer in BRCA1 and BRCA2 mutation carriers. J Natl Cancer Inst 2002; 94: 1773 1779.

3. Jernstrom HC, Olsson H, Borg A. Reduced testosterone, 17 beta-oestradiol and sexual hormone binding globulin, and increased insulin-like growth factor- 1 concentrations, in healthy nulligravid women aged 19-25 years who were first and/or second degree relatives to breast cancer patients. Eur J Cancer Prev 1997; 6: 330-340. 
4. Brunet JS, Ghadirian P, Rebbeck TR, Lerman C, Garber JE, Tonin PN, Abrahamson J, Foulkes WD, Daly M, Wagner-Costalas J, Godwin A, Olopade OI, Moslehi R, Liede A, Futreal PA, Weber BL, Lenoir GM, Lynch HT, Narod SA. Effect of smoking on breast cancer in carriers of mutant BRCA1 or BRCA2 genes. J Natl Cancer Inst 1998; 90: 761-766.

5. Gayther SA, Mangion J, Russell P, Seal S, Barfoot R, Ponder BA, Stratton MR, Easton D. Variation of risks of breast and ovarian cancer associated with different germline mutations of the BRCA2 gene. Nat Genet 1997; 15: 103-105.

6. Thompson D, Easton DF. Breast Cancer Linkage Consortium. Cancer Incidence in BRCA1 mutation carriers. J Natl Cancer Inst 2002; 94: 1358-1365.

7. Easton DF, Ford D, Bishop DT. Breast Cancer Linkage Consortium. Breast and ovarian cancer incidence in BRCA 1 -mutation carriers. Am J Hum Genet. 1995; 56: 265-271.

8. Moller P, Heimdal K, Apold J. Norwegian Inherited Breast Cancer Group; Norwegian Inherited Ovarian Cancer Group. Genetic epidemiology of BRCA1 mutations in Norway. Eur J Cancer 2001; 37: 2428-2434.

9. Heimdal K, Maehle L, Apold J. The Norwegian founder mutations in BRCA1: high penetrance confirmed in an incident cancer series and differences observed in the risk of ovarian cancer. Eur J Cancer 2003; 39: 2205-2213.

10. Bodd TL, Reichelt J, Heimdal K, Moller P. Uptake of BRCAI genetic testing in adult sisters and daughters of known mutation carriers in Norway. J Genet Couns 2003; 12: 405-417.

11. Antoniou AC, Pharoah PD, McMullan G. Evidence for further breast cancer susceptibility genes in addition to BRCA1 and BRCA2 in a population-based study. Genet Epidemiol 2001; 21: 1-18.

12. Antoniou AC, Gayther SA, Stratton JF et al. Risk models for familial ovarian and breast cancer. Genet Epidemiol 2000; 18: 173-190. 